\title{
Mitochondrial Dysfunction in Neurodegenerative Dis- orders and Atherosclerosis: Pathophysiological Mecha- nisms and innovative Protocol Towards an Elegant New Era Of Health
}

\author{
Abdullah Abdulrhman Al Abdulgader* \\ Senior Congenital Cardiologist and Invasive Electrophysiologist, Prince Sultan Cardiac Center (Founder\& Chief Physician), Saudi Arabia
}

*Corresponding author: Abdullah Abdulrhman Al Abdulgader, Senior Congenital Cardiologist and Invasive Electrophysiologist, Prince Sultan Cardiac Center (Founder\& Chief Physician), Alhasa, Saudi Arabia

To Cite This Article: Abdullah Abdulrhman Al Abdulgader, Mitochondrial Dysfunction in Neurodegenerative Disorders and Atherosclerosis: Pathophysiological Mechanisms and innovative Protocol Towards an Elegant New Era Of Health. 2020 - 11(1). AJBSR.MS.ID.001582.

DOI: 10.34297/AJBSR.2020.11.001582.

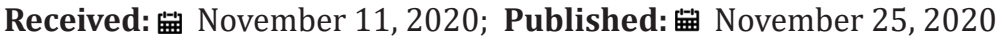

\section{Introduction}

The energy plants of the human being are composed of trillions of mitochondria which is semi-autonomous, double membranebound cellular organelles that is in charge of generating most of the chemical energy needed to operate the cellular biochemical reactions in the human body. The sizes of mitochondria vary from 0.75 to $8 \mathrm{~mm}$ depending on the cell type, cell cycle stage, and intracellular metabolic state. These organelles make up as much as $10 \%$ of the cell volume but responsible of producing $90 \%$ of the human needs of energy. Mitochondria can be divided into four functional areas, the mitochondrial outer membrane (OMM), mitochondrial membrane space, mitochondrial inner membrane (IMM), and mitochondrial matrix. The IMM is rich with various membrane proteins and the location where adenosine triphosphate ATP is generated via the proton motive force produced by the electron transport chain. Chemical energy produced by the mitochondria is stored in ATP. Mitochondrion (pleural is mitochondria) is found in every cell of the human body except red blood cells. Mitochondria contain their own small chromosomes. Mitochondrial DNA, are inherited only from the mother. Due to its ubiquitous presence in the body systems, its dysfunction is expected to be expressed in diverse symptoms and complaints of the heart brain ,kidney, pancreas, ears, eye, nerves ,muscles and other systemic manifestations. Two major pathologic disease mechanisms contributing to vast degenerative diseases in human, namely, neurodegenerative (ND) disorders and atherosclerosis are thought to have underlying mi tochondrial dysfunction as major contributing pathological mechanism. ND disorders like Alzheimer's disease(AD), Huntington's disease(HD), Parkinson's disease(PD), Amyotrophic lateral sclerosis(ALS), Epilepsy, Schizophrenia, Multiple sclerosis(MS), and Neuropathic pain involves mitochondrial dysfunction and is regarded as the core of their pathological processes. Apoptosis is regulated by mitochondria and thought to be of central role in the process of ND disorders and atherosclerosis. Altered signaling of the apoptotic mechanisms are evident in neurodegeneration. The pathogenesis of neurological disorders is promoted by abnormal levels of molecular apoptotic proteins. Mitochondrial dysfunction yields higher levels of reactive oxygen species (ROS). Non-apoptotic death of cells is initiated by raised ROS levels. ROS produced in cells acts as signaling molecules, but when produced in larger amounts will result in cellular consequences to deoxyribonucleic acid, proteins and lipids, decreased effectiveness of cellular mechanisms, that will promote inflammatory pathways, promote neuronal death caused by hyperactivity of excitatory amino acids -mainly Glutamate (Glu)-in the mammal Central Nervous System (excitotoxicity), protein agglomeration and apoptosis. Increased production of reactive oxygen species in mitochondria, accumulation of mitochondrial DNA damage, and progressive respiratory chain dysfunction are associated with atherosclerosis or cardiomyopathy in human and animal models. Other predisposing factors to atherosclerosis like, hyperglycemia, 
hypertriglyceridemia, and aging can induce mitochondrial dysfunction. Another indirect promoters of atherosclerosis including chronic overproduction of mitochondrial reactive oxygen species which can destroy pancreatic cells, increased oxidation of low-density lipoprotein and dysfunction of endothelial cells. Recent scientific literature disclosing new discoveries illustrating that mitochondria are highly dynamic organelles even,beyond powerhouses of a cell that play important roles in cell homeostasis by regulating cell function and phenotypic modulation. In this original research article, up to date pathophysiological processes involved in mitochondrial dysfunction and its impact in the different ND disorders and atherosclerosis will be discussed. An innovative unique approach targeting mitochondrial apoptotic pathways and oxidative phosphorylation defects and other vital mitochondrial dysfunction consequences is discussed carrying new will toward ameliorating and/or aborting the process of atherosclerosis and neurodegeneration in human species.

\section{Mitochondrial dynamics in health and disease}

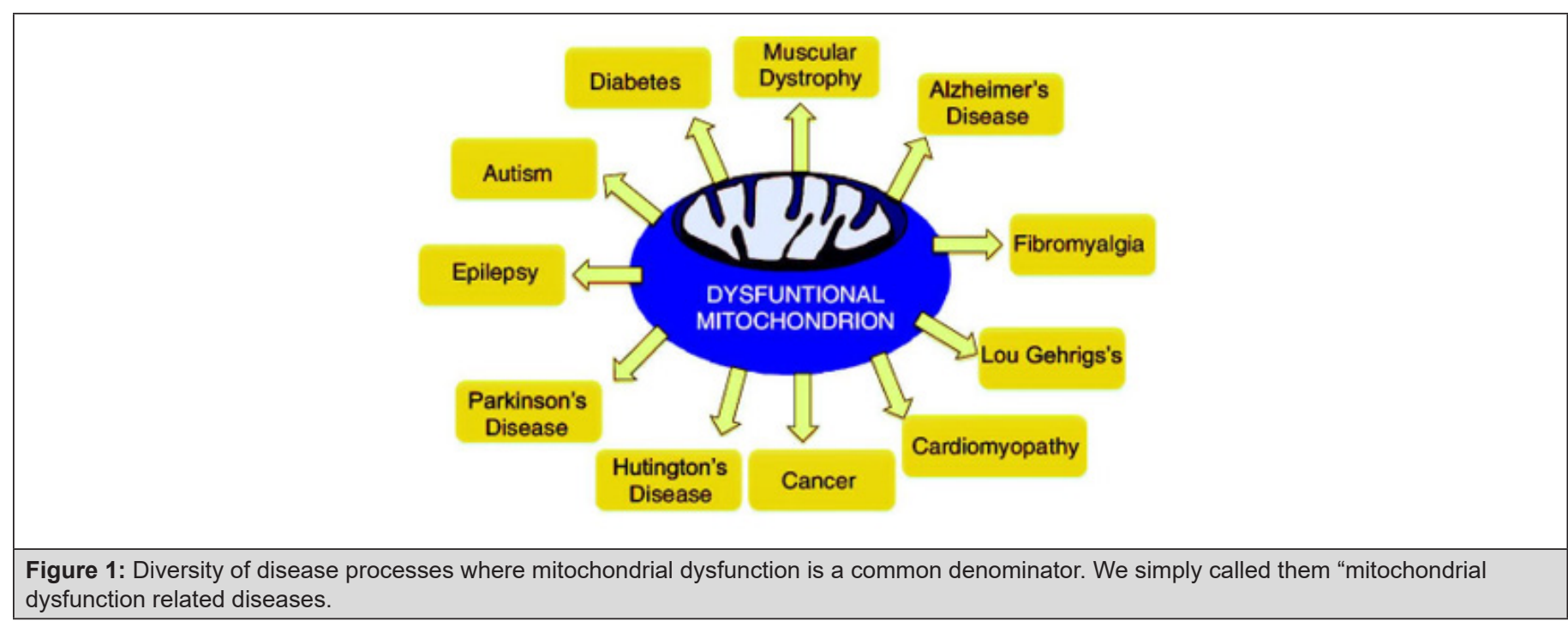

Mitochondrial diseases are a heterogeneous group of disorders with varying clinical features caused by impaired function of the mitochondrial respiratory chain. The underlying cause can be genetic or environmental. Gene mutations affecting either the nuclear DNA or the mitochondrial DNA (mtDNA), both of which contain genes encoding components of the oxidative phosphorylation machinery required to generate ATP, the currency of cellular energy [1]. Spectrum of more than 250 distinct mitochondrial diseases have been linked to a variety of pathogenic variants occurring in the nuclear or mitochondrial genomes; these diseases typically manifest as severe and often lethal multisystemic disorders [2]. Mitochondrial dysfunctions involved in pathophysiology of neuropsychiatric disorders include disturbances in oxidative phosphorylation(OXPHOS), increased mitochondrial DNA (mtDNA) deletions, mutations or polymorphisms, impaired calcium signalling, and impaired energy metabolism as well as interactions with disease specific proteins (e.g., $\beta$ Amyloid peptide (A $\beta$ ), parkin,PINK1, alpha-synuclein, and huntingtin) [3] (Figure 1).

Mitochondrial trafficking which implies that mitochondria are dynamic organelles that are transported on cytoskeletal proteins is a feature of this very critical cellular organelle. Part of the normal mitochondrial behavior that is happening constantly and in a wellorganized manner is fusion (mediated by OPA1, Mfn1, and Mfn2) and fission(means division) (proteins fission 1 and Drp1), swell, extend, fragment, and are recycling (mitophagy or vesicle formation) . Fusion disorganization leads to mitochondrial elongation, and unbalanced fission will end up with excessive mitochondrial fragmentation and small mitochondria, both of which impair the function of mitochondria. Mitochondrial function and organelle distribution in neurons require efficient exchange of mitochondrial contents. Mitochondrial fusion, in particular, is required for proper development and maintenance of the cerebellum, is mediated in particular by Mfn2 [4]. Charcot-Marie-Tooth type 2A is caused by Mutations in the Mfn2 gene. Dominantly inherited optic atrophy is caused by mutations in OPA1. Scientific evidence is in rise documenting cause effect relationship of altered mitochondrial trafficking and fusion-fission dynamics and constellation of ND disorders including Alzheimer's disease (AD), Parkinson's disease (PD), Huntington's disease (HD), and amyotrophic lateral sclerosis (ALS).Mitochondrial dysfunction contribute to atherosclerosis with different pathologic mechanisms including: mitochondrial DNA Damage, endothelial dysfunction, smooth muscle cells related mechanisms and macrophage activation derangements. Apoptosis, calcium signaling, proliferation, inflammation, and reactive oxygen species (ROS) synthesis are considered to be non power production functions of mitochondria that might contribute to ND and atherosclerosis. 


\section{Mitochondrial Dysfunction and Neurodegenerative Disorders}

\section{Clinical and histopathological findings}

Alzheimer's disease(AD) is the most common ND disorder.AD is characterized by progressive cognitive decline usually starting with impairment in the ability to form recent memories, progressing to affect all intellectual functions and ending up with complete dependence of basic life activities and ultimately , premature death. This trajec clinical deterioration is rooted to histopathological changes in the form of extracellular neuritic plaques and intracellular neurofibrillary tangles. Most of the plaque is amyloid peptide, while the tangles is composed of hyperphosphorylated tau proteins. It has been proposed that in sporadic AD mitochondrial dysfunction is the primary event that causes Amyloid peptide deposition, synaptic degeneration, and formation of neurofibrillary tangles [5]. AD brains and peripheral cells derived from patients with $\mathrm{AD}$ are characterized by Energy deficiency [6,7]. Strategic three enzymatic systems in energy production are impaired in postmortem $\mathrm{AD}$ brain and fibroblasts, namely, tricarboxylic acid cycle enzyme complexes, pyruvate dehydrogenase, isocitrate dehydrogenase, and alpha-ketoglutarate dehydrogenase [8]. Platelets and lymphocytes OXPHOS complex I, III, and IV activities from patients with postmortem $\mathrm{AD}$ brain tissue were found to be reduced $[9,10]$. Mitochondrial biogenesis is also deficient [11].

Parkinson's disease (PD) is the second most common neurodegenerative disorder. Triad of resting tremor, bradykinesia, and rigidity due to neurodegeneration and loss of dopaminergic (DA) neurons are the clinical hallmarks of PD. Loss of pigmented dopaminergic neurons in the substantia nigra and the presence of abnormal protein aggregates called Lewy bodies are the pathological hallkark of PD. Lewy bodies are cytoplasmic eosinophilic inclusions composed of the presynaptic protein alpha -synuclein. The heaviness of evidence pointing to mitochondrial dysfunction as abases for PD is accumulating in the last 14 years. Rotenone and 1-methyl-4-phenyl-1,2,3,6-tetrahydropyridine (MPTP) when used as inhibitors for OXPHOS complex, in animal models, will produce neuropathologic and behavioral symptoms similar to human PD. Group of proteins PD including : phosphatase and tensin homolog-induced putative kinase 1 (PINK1), DJ-1, alpha-synuclein, leucine-rich repeat kinase 2 , and parkin, are genetically linked to familial PD.Those proteins are either mitochondrial proteins or are associated with mitochondria. In specific Parkin and PINK1 have a direct role in the cell's mitochondrial quality-control pathways where they have role in identifying impaired mitochondria with reduced membrane potential and selectively eliminating them from the mitochondrial network by mitophagy. Failure of mitophagy is thought to play a role in the pathogenesis of PD [12]. The review of today's medical literature will illustrate the fact that different neurodegenerative disorders and other mitochondrial dysfunctions affecting different systems have common pathways and similarities. It seems that specific trigger in specific cell type (AD: neuron microglia, PD: neuron ganglia, HD: neuron astrocyte, ALS: neuron) with fine tuning of certain mechanism/s in different body systems will favor the production of specific disorder rather than other mitochondrial dysfunction disorders. Figure 2 illustrate the possible defective mechanisms in PD as a model for other ND disorders and mitochondrial dysfunction in general.

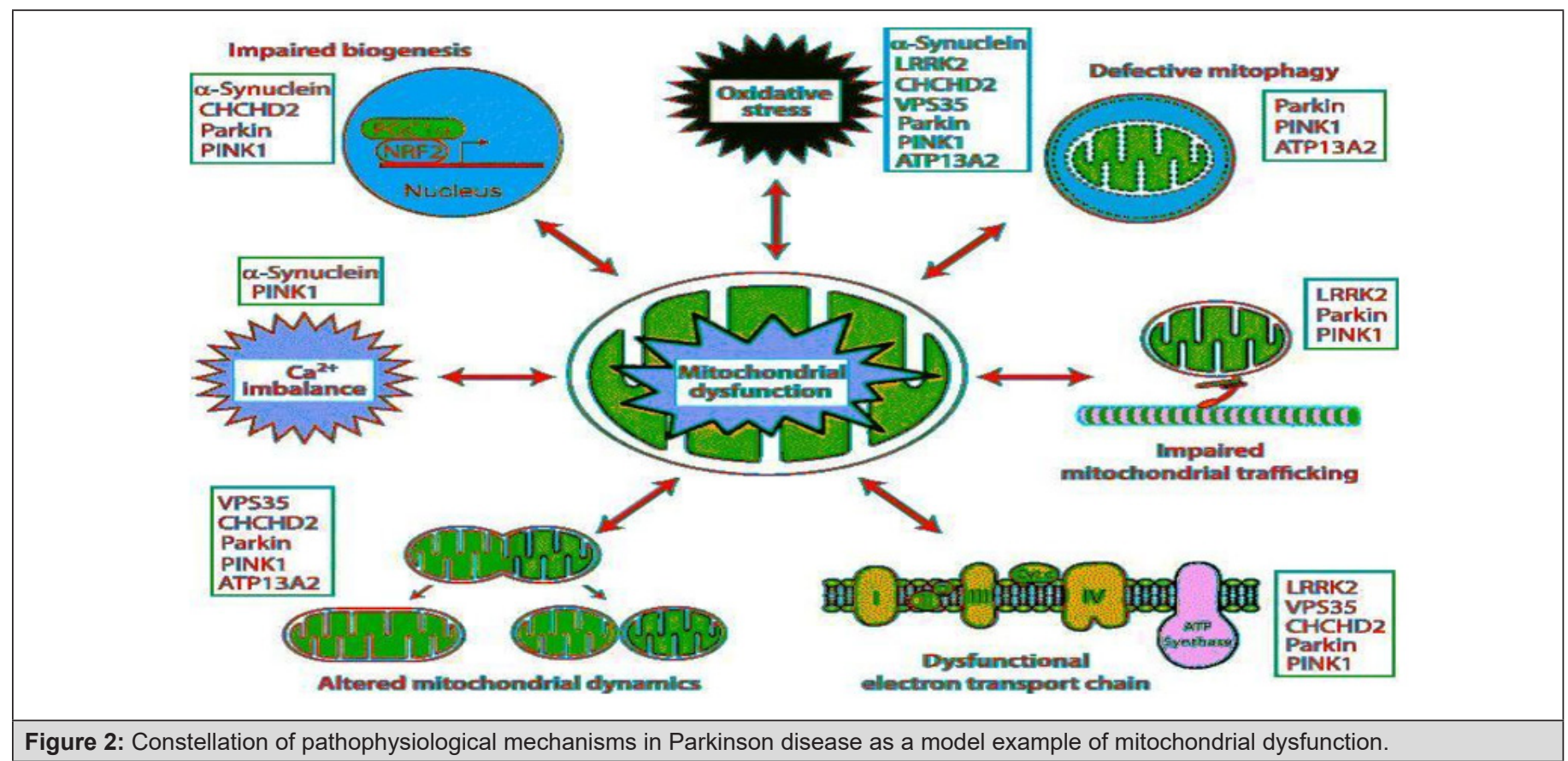


Defective mechanism may operate in bidirectional pathways and affects mitochondrial membrane permability, trafficking, mitophagy, biogenesis, calcium imbalance and the different mutations resulting in defective mitochondria operations. Those mechanisms may act as single defect or in combination with resultant increase in ROS and cell apoptosis. The complex interplay of the different defective mechanisms may end up with vicious cycle resulting in cell dysfunction and/or death. Huntington's disease (HD) is a dominantly inherited progressive neurodegenerative disease, caused by $\geq 36 \mathrm{CAG}$ repeats in the HD gene (htt). Approximately $10 \%$ of patients inherit a chromosome that underwent CAG expansion from an unaffected parent with $<36$ CAG repeats [13]. The disease is characterized by progressive motor impairment, personality changes, psychiatric illness, and gradual intellectual decline. Pathologically, there is a preferential and progressive loss of the medium spiny neurons in the striatum, as well as cortical atrophy, and degeneration of other brain regions later in the disease. Bioenergetic deficiency and mitochondrial dysfunction is a hallmark in HD as documented by extensive evidence in recent years medical literature. Bioenergetic deficiencies is evident by constellation of clinical and laboratory findings. Pronounced weight loss despite sustained caloric intake, nuclear magnetic resonance spectroscopy showing increased lactate in the cerebral cortex and basal ganglia, decreased activities of OXPHOS complexes II and III, reduced aconitase activity in the basal ganglia, abnormal mitochondrial membrane depolarization in patient lymphoblasts, abnormal ultrastructure of mitochondria in cortical biopsies obtained from patients with both juvenile and adult-onset HD, and pathologic-grade dependent reductions in numbers of mitochondria in HD postmortem brain tissue and in striatal cells [14]. Mitochondrial respiration and ATP production are significantly impaired in mice models [15].

Amyotrophic lateral sclerosis (ALS), also known as Lou Gehrig's disease, in Canada and the U.S., and as motor neurone disease (MND) in the UK and Australia, is a neurodegenerative neuromuscular disease that results in the progressive loss of motor neurons that control voluntary muscles. It is the most common type of motor neuron disease. Mitochondrial dysfunction has been implicated as playing a role in motor neuron death in ALS. Fragmentation of mitochondria and changes in mitochondrial morphology and expression of fusion/fission proteins are well described in ALS and have pronounced effects on normal mitochondrial function [16]. Accumulation of abnormal mitochondria in motor neuron axons seen in animal models of ALS and also in human patients might be due to defective mitochondrial transport. Aberrations in oxidative metabolism linked to changes in electron transport chain (ETC) activity and impaired ATP production was shown in cell culture models and in transgenic animals [17-20]. Impaired Ca2+ homeostasis and an increased production of reactive oxygen species (ROS) which is associated with oxidative-related damage including changes in protein carbonylates and tyrosine nitration are seen in mitochondria from ALS [21,22]. Glutamate-receptor mediated neurotoxicity has been linked to an overload of mitochondrial calcium and ROS production in cultured spinal motor neurons from transgenic ALS animals [23]. Today medical literature is documenting mitochondrial dysfunction and impaired dynamics are a central and common feature of the pathogenesis in ALS. Inspite of all studies we reviewed, we cannot judge if mitochondrial dysfunction is a primary or secondary process in ALS. Mutant SOD1 in animal models, typically display a phenotype that resembles ALS and demonstrate most of the histopathological and biochemical features and also the symptoms of the human disease.

\section{Neurodegeneration secondary to mitochondrial DNA mutations and peroxisome dysfunction}

Mitochondria are characterized by their own DNA (mtDNA). Thirteen out of the 92 polypeptides of the OXPHOS system are encoded in mtDNA ,the remaining structural polypeptides and assembly factors are encoded by nuclear DNA. Dysfunction of OXPHOS can be due to mutations in either mtDNA or nuclear DNA. This type of cellular dysfunction is well known to affect tissues with high energy demands such as the heart, central nervous system, and skeletal muscle. Aging related changes are thought to be through the accumulation of mtDNA mutations and net production of ROS. Mitochondrial DNA mutations, mitochondrial abnormalities, and mitochondrial respiratory chain-deficient cells are also present in age-related neurodegenerative diseases such as PD and AD [24-29]. Peroxisome proliferator-activated receptor (PPAR) gamma coactivator (PGC)-1 alpha is a transcriptional coactivator has wide spread role in cellular vital events. It interacts with a broad range of transcription factors involved in a wide variety of biological processes and/or responses. Its vital functions include mitochondrial biogenesis, OXPHOS, antioxidant defense, adaptive thermogenesis, glucose/fatty acid metabolism, fiber type switching in skeletal muscle, and heart development [30,31]. Recent literature in the subject confirm the pathologic role of PGC-1 alpha expression and/or function impairment as a common underlying cause of mitochondrial dysfunction in neurodegenerative diseases such as HD, PD, and AD.

\section{Mitochondrial dysfunction in atherosclerosis}

Coronary heart disease still rank number one killer of all world nations. Inspite of the explosive scientific advances of the 20th century and after, it is now conspicuous that sincere call for wisdom with new basic understanding of coronary heart disease, is highly needed [10,32]. Recent medical literature have shown that mitochondrial dysfunction contributing to ND disorders-discussed earlier- with specific derangement is also contributor to atherosclerosis in human. Many studies suggested that mitochondrial $\mathrm{K}+$ channels have an important role in cardioprotection. The mechanism of cardioprotection, 
though unclear, may involve mild uncoupling of the mitochondrial membrane potential causing $\mathrm{Ca} 2+$ overload and ROS generation.

\section{Mitochondrial DNA Damage in atherosclerosis}

The human mtDNA is a 16,569-bp circular double-stranded molecule, which attaches to the IMM. The mtDNA encodes 37 genes, including 13 proteins, 2 ribosomal RNA (rRNA), and 22 transfer RNA (tRNA). These 13 proteins are subunits of respiratory complexes I, III, IV, and V with essential roles in oxidative phosphorylation (OXPHOS). The mtDNA-derived rRNA and tRNA are required for protein synthesis in the mitochondria. The mtDNA owns no defense against damage [33]. This is due to the fact that mtDNA lack of introns and histone protection as well as absence of effective DNA damage repair system. Mitochondrial DNA located in close proximity to ROS production site. Accordingly, it can be attacked easily by ROS. Proofreading capability is lacking in the DNA polymerase of mitochondria, leading to a high error rate during the replication of mtDNA. In addition, the mtDNA is continuously undergoing synthesis in the entire cycle, leading to poor stability and susceptibility to external interaction. Those factors collectively will lead to high mutation rates in mtDNA $[34,35]$. Mutations of the mtDNA will result in reduction of the formation of transmembrane proton electrochemical gradient, the synthesis of ATP energy species, and the ability of cells to maintain ATP/ADP ratios with reduction of ATP biosynthesis. Mitochondrial gene mutations and cardiovascular disease has been shown to be closely related to hypertension, primary cardiomyopathy, atrial fibrillation, heart failure, erogenesis, and atherosclerosis by regulating systemic metabolism inflammation, proliferation, and apoptosis [36]. The mitochondrial dysfunction causes a short opening of mitochondrial permeability transition pore (MPTP) and depolarization of mitochondrial membrane potential. MPTP was originally discovered by Haworth and Hunter in 1979. It is a protein that is formed in the inner membrane of the mitochondria under certain pathological conditions. MPTP opening allows increase in the permeability of the mitochondrial membranes to molecules of less than 1500 Daltons in molecular weight. The prolonged opening of the MPTP leads to the expansion of mitochondrial matrix and rupture of the outer membrane. This process results in the release of pro-apoptotic factors in the membrane gap and cell apoptosis [37]. Impaired mitochondrial function, is a casual factor leading to abnormal proliferation of vascular smooth muscle cells (VSMCs) and excessive apoptosis and release of inflammatory factors. These phenomena promoted the development of atherosclerosis and plaque vulnerability [38].

\section{Reactive oxygen species (ROS) and atherosclerosis}

The main way of cellular energy production is through the mitochondrial respiratory chain. In physiological environment, the respiratory chain is efficient using over $98 \%$ of the electron transport for ATP synthesis. The remaining 1-2\% of electrons are released to generate superoxide radical, such as intracellular ROS . In general, ROS is essential for cellular function, and excess ROS is cleared by antioxidant defense. Physiological level of superoxide is produced by OXPHOS of the mitochondria which is converted to hydrogen peroxide by the enzyme manganese-dependent superoxide dismutase (SOD2) followed by another enzyme, glutathione peroxidase 1 (GPx1) into water [39]. Under pathophysiological conditions, chronic and acute overproduction of ROS is released from different sources, such as xanthine oxidase, lipoxygenase, and nicotinamide adenine dinucleotide phosphate oxidase, and the uncoupling of nitric oxide (NO) synthase, especially the leakage of electrons at complex I and complex III during OXPHOS [40]. "ROSinduced ROS" Is the description given to the production of large amount of ROS from mitochondria induced by defective adjacent mitochondria. Physiologically excessive ROS production will stimulate positive feedback mechanism which in turn amplify the ROS signal and further induces mitochondria and cell dysfunction. Antioxidants, such as glutathione, carotenoids, catalase, and glutathione peroxidase will counteract excessive ROS production. When this balance is destroyed, the oxidative modification in the arterial wall is increased [41]. These phenomena are associated with increased mitochondrial ROS and the progression of atherosclerosis [42] Mitochondrion-derived ROS act as signal transduction molecules, and they trigger the upregulation of inflammatory cytokine subpopulations through different molecular pathways [41]. Excessive production of ROS will result in DNA fragmentation and monocytes apoptosis in normocholesterolemic old mice. This denotes that increased ROS promoted the aggravation of age-related atherosclerosis [43]. Poly (ADPribose) polymerase 1 (PARP1) production is enhanced by ROS, which led to excessive DNA damage and death of VSMCs and vascular endothelial cells (VECs) [44]. Besides, the impaired mitochondrial electron transport chain results in the overproduction of ROS, which leads to the oxidation of lipids and proteins and extensive cellular damage. This enhanced ROS production induces endothelial dysfunction and vascular inflammation, in the arterial wall, which contribute to the formation of initial lesions and their maturation of late plaques and possibly progression to plaque rupture. Given the critical role of ROS in the pathogenesis of atherosclerosis, the effective removal of ROS may be an efficient measure for the prevention and treatment of atherosclerosis. A systemic administration of mitochondriatargeted antioxidant Mitoquinone (MitoQ) reduced atherosclerotic lesion macrophage accumulation and Smooth Muscle Cell proliferation in fat-fed Apolipoprotein E (APOE) mice [45]. ApoE is the principal cholesterol carrier in the brain. However, vit $\mathrm{E}$ was not found to be effective to prevent coronary heart disease. In addition, other antioxidants, such as Glutaredoxin-2a and SOD1/3, have no obvious effect in reducing atherosclerotic lesions [46]. The role of antioxidants in atherosclerosis is still controversial and needs to be more investigated. Specific cellular pathways to interfere 
with atherosclerosis in humans will be the subject of upcoming discussion in this research article.

\section{Atherosclerosis, and mitochondria related endothelial dysfunction}

Vascular homeostasis is the role of the endothelium. Endothelial cells are doing this through regulating vasodilation, platelet activation, leukocyte adhesion, and smooth muscle cell proliferation and migration. Endothelial dysfunction is the earliest pathophysiological change of atherosclerosis, the whole mark of which is reduction of NO synthesis and secretion $[47,48]$. Endothelial NO plays an important anti-inflammatory and antithrombotic role through prevention of the expression of endothelial cell adhesion molecules and chemokines, and inhibition of platelet activation and aggregation [49]. Endothelial nitric oxide synthase(eNOS) catalyze the conversion of L-arginine into L-citrulline to yield NO. NO biosynthesis demands the availability of NADPH, flavin mononucleotide, flavin adenine dinucleotide, tetrahydrobiopterin, and $\mathrm{Ca} 2+/$ calmodulin $[48,49]$. Endothelial nitric oxide synthase (eNOS) degradation due to ROS-mediated oxidative stress, is the main reason for reduction of NO production. More ROS is produced by eNOS decoupling dysfunction, resulting in more damage of endothelial function a process which ultimately, promoting the development of atherosclerosis [50]. The mitochondria play a key role in Extra Cellular function as factors that cause several processes such as nitric acid production, apoptosis, and intracellular signaling. Extra Cellular senescence, apoptosis, and development of atherosclerosis are all sequel of Excessive ROS production [41,51]. Endothelial cell dysfunction with mitochondrial damage and dysfunction precedes the development of cardiovascular disease [52]. Activated neutrophils is shown to accumulate in areas of endothelial injury and generated large amounts of ROS during the process of atherosclerosis and ischemic injury [53,54]. ROS bind to NO to yield peroxynitrite (ONOO-). Peroxynitrite is an oxidant and nitrating agent. Due to its oxidizing properties, peroxynitrite can damage a wide array of molecules in cells, including DNA and proteins. Peroxynitrite inhibits mitochondrial protein synthesis in a dose-dependent manner and results in decreased cellular ATP levels and mitochondrial reduction [55]. Peroxynitrite affect the uncoupling of eNOS leading to 02 to generation but $\mathrm{NO}$ is not synthesized any more. On the other hand, ROS promote eNOS uncoupling a process that will lead to more ROS production in a vicious cycle manner that will end up with profound damage to mitochondria endothelial function [56].

\section{Peroxisome and mitochondrial dysfunction}

Peroxisomes are oxidative organelles that owe their name to hydrogen peroxide generating and scavenging activities. Studies have shown that Peroxisome proliferator-activated receptor (PPAR) gamma coactivator (PGC)-1 alpha is- as pointed earlier- clearly a key regulator of mitochondrial biosynthesis. The mitochondrial transcript factor $\mathrm{A}$ and transcription factor $\mathrm{B}$, which regulate the expression of genes encoded by mtDNA, including the essential subunits of the electron transport chain, is activated by PGC-1alpha [57]. PGC-1 alpha restores the function of NO and significantly inhibits the damage of endothelium-dependent vasodilatation induced by oxidized fatty acids. Accordingly, PGC-1alpha restored endothelial cell fatty acid oxidization, leading to increase ATP synthesis [58]. This will result in normalization of mitochondrial membrane potential and ROS production. The overexpression of PGC-1alpha under shear stress promotes NO production and mitochondrial biogenesis, while NO and PGC-1alpha independently counteract ROS production, thereby limiting endothelial dysfunction. Oxidized LDL promotes inflammation, but PGC-1alpha blocks oxidized LDL from entering the cells, and accordingly prevent inflammation pathways activation. PGC-1a also regulates vascular endothelial growth factor-1 expression and stimulates angiogenesis [48]. The inhibition of the expression of PGC-1 alpha significantly decreases mitochondrial biosynthesis in vascular endothelial cells. ApoE -/- PGC-1a -/- mice showed increased inflammatory factor expression [59]. Therefore, the overexpression of PGC-1alpha prevents apoptosis, restricts inflammatory activation, prevents cJun N-terminal kinase activation, and increases NO bioavailability [57]. c- Jun N-terminal kinase belongs to the mitogen-activated protein kinase family, and are responsive to stress stimuli, such as cytokines, ultraviolet irradiation, heat shock, and osmotic shock. Increasing the expression of PGC-1alpha in vascular endothelial cells or ATP/ADP translocating enzyme activity may prevent or treat atherosclerosis. The percentage of mitochondria from cytoplasm volume is cell type specific. Endothelial cells have mitochondrial content of only $2-6 \%$ of the cytoplasm volume, compared to the blood-brain barrier mitochondrial content which may reach 8- 11\% [60]. In addition to mitochondrial critical role as energy plant and reservoir, it orchestrate sensing function of blood oxygen levels and transmit information to cardiomyocytes and regulate NO-mediated vasodilation $[61,62]$.

\section{Vascular smooth muscle cells(VSMCs) and Mitochondrial dynamics}

VSMCs are the main components of the blood vessel wall as well as plaques. In physiological conditions, VSMCs regulate blood flow and pressure by regulating blood vessel tension with contractile phenotype [63]. A series of contractile proteins, such as SM a-actin (SMaA), SM-22a, SM myosin heavy chains SM-1 and SM-2, calponin, and smoothelin are expressed by these contractile cells. The phenotype will change from contractile to synthetic under mechanical damage or biostimulation in a process called dedifferentiation. Synthetic VSMCs downregulate contractile protein expression, increase proliferation, and remodel extracellular matrix (ECM) to promote migration. These phenomena lead to 
functional disorders, thickening of the wall, narrowing of lumen, decreased vascular compliance, and vascular remodeling, with exacerbation of atherosclerosis (63). VSMCs differentiation status changes contribute to the pathogenesis of various cardiovascular diseases, such as atherosclerosis, hypertension, and vascular stenosis. Defective mitochondria with impaired OXPHOS is well known pathological finding of SMCs isolated from human atherosclerotic lesions [64,65]. Damaged VSMCs' nuclear DNA and mtDNA in atherosclerotic patients demands higher amount of ATP, active substances, and oxygen-free radicals. This phenomenon will exacerbate more DNA damage, genomic instability, and mitochondrial dysfunction.

\section{Macrophage activation and mitochondrial dysfunction}

Macrophages are the most abundant cell type in atherosclerotic Plque and known to be critical for atherosclerotic process progression. Macrophage has striking plasticity as it bears a polarized phenotype with specific functional characteristic responses to local microenvironmental stimuli, [66]. Macrophages are subdivided into M1 which is classically activated macrophages and M2 which is alternatively activated macrophages. M1 macrophages enhance inflammation, while M2 macrophages is known to halt inflammation and repair tissue damage. Compared with M1macrophages, M2 macrophages showed lower cholesterol uptake and lipid accumulation [67,68] Atherosclerosis is a pathological process characterized by chronic inflammatory reactions, and its development is always accompanied by an inflammatory reaction. Several other macrophage subpopulations were also observed in atherosclerotic plaques. During atherosclerotic process, both M1 and M2 macrophage numbers are increasing and mainly distributed at the fibrous cap region. M1 macrophage is the major macrophage subtype in unstable human plaques with large lipid core, whereas M2 is abundantly found in stable plaques. A therosclerotic plaque progression and instability is affected by the dynamics of macrophage polarization and the proportion of these macrophages. Mitochondrial oxidative metabolism contributed to macrophage alternative activation while glycolysis is the main energy source of M1 macrophages, whereas mitochondrial OXPHOS is the major energy source of M2 macrophages [70]. M1 activation inhibits mitochondrial function and reduces mitochondrial oxygen consumption, indicating mitochondrial dysfunction in M1 cells. Mitochondrial function is required for the induction and maintenance of M2 phenotype [71]. Macrophage mitochondrial ROS above physiological levels can induce its polarization to M1. M1 macrophages have strong phagocytosis and bactericidal capacity and can produce a large number of proinflammatory cytokines, such as TNF, IL-1, IL-6, IL12, IL-18, IL-23, and Nitrous Oxide Systems(NOS) (72). Moreover, damaged mtDNA binds to NLR family pyrin domain containing 3 (NLRP3) to form a complex and is associated with apoptosis- associated speck-like protein (ASC). Then, the damaged mtDNA binds to caspase- 1 precursor and forms an inflammasome(which is cytosolic multiprotein oligomers of the innate immune system responsible for the activation of inflammatory response), and this process promotes the production of mitochondrial ROS. Thus, inflammatory factors are secreted, and the initiation and progression of atherosclerosis is promoted. A unique macrophage phenotype Mhem, a hemoglobin driven macrophage phenotype, is found in the area of hemorrhage in human atherosclerotic plaques, which limits the progression of atherosclerotic lesions by increasing HO-1 expression, inhibiting inflammation, and increasing the secretion of IL-10 [73]. As a matter of fact, the mechanisms controlling the generation of these different subtypes, together with their bioenergetics and metabolic profiles, are poorly understood [33].

Towards Innovative Therapies for Mitochondrial Dysfunction in Atherosclerosis and Neurodegenerative Diseases

\section{Alabdulgader, K.McCully SAHACT nutritional-metabolic protocol to combat mitochondrial dysfunction}

The discovery of abnormal homocysteine metabolism in arteriosclerosis in 1969 and in cancer in 1976 suggests a novel approach to prevention and treatment of these important degenerative diseases of aging in susceptible populations [74]. Since publication of these discoveries, recent new insights into the function of homocysteine in cellular metabolism have pointed to the importance of nutritional and metabolic abnormalities of homocysteine metabolism in causing arteriosclerosis and degenerative diseases. Obstruction of vasa vasorum by aggregates of microorganisms with homocysteinylated low-density lipoproteins is proposed to cause ischemia of arterial wall and a micro abscess of the intima, the vulnerable atherosclerotic plaque [74]. Experimental work by Kimer McCully and others demonstrate excitotoxicity, apoptosis, and mitochondrial oxidative stress induced by homocysteine. Those results support excitotoxicity induced by homocysteine as an important factor in the apoptosis observed in atherogenesis and the neuronal necrosis observed in neurodegenerative diseases, including dementia [75], schizophrenia [76], cognitive decline [77], and multiple sclerosis [78]. An innovative protocol based on these discoveries has been adopted by our team pioneered by the father of homocystein theory in modern medicine, Kilmer McCully in the multicenter metabolic nutritional project known as: Saudi Arabia Homocysteine Arteriosclerosis Cancer Trial (SAHACT). The expected results of the SAHACT trial will demonstrate the efficacy of the nutritional-metabolic approach to control of degenerative diseases of aging, in direct comparison with current methods. The SAHACT trial utilizes an innovative approach to control of the most important diseases of aging populations in Saudi Arabia and other countries. 
The strategic technologies addressed in the SAHACT trial relate to new understanding of the abnormal homocysteine metabolism underlying degenerative diseases. Novel therapeutic molecules which target mitochondrial function and excessive ROS production implicated in the progression of atherosclerosis, are immediately needed for the wellbeing of human medical communities in this era. The tracks of new technologies include catabolism of homocysteinylated macromolecules, biosynthesis of thioretinaco from retinol, thioretinamide and cobalamin, catabolism of homocysteine by cystathionine synthase, formation of transthyretin from essential amino acids, control of homocysteine metabolism by beneficial dietary fats and proteins, and antibiotics to control associated infections. New understanding of the importance of retinol (vitamin A), ascorbate (vitamin C), and homocysteine thiolactone in the biosynthesis of thioretinamide and thioretinaco by cystathionine synthase suggests a promising method for control of abnormal homocysteine metabolism in human subjects with arteriosclerosis and neurodegeneration. The recent advances and knowledge of mitochondrial functions and dysfunctions were incorporated intelligently in our protocol. The efficacy of pancreatic enzyme therapy of cancer is interpreted as a promising method for promoting catabolism of macromolecules, specifically proteins, nucleic acids, and glycosaminoglycans that contain excess homocysteine groups resulting from abnormal accumulation of homocysteine thiolactone in aging, atherogenesis ,carcinogenesis and neurodegenerative disorders. Dietary deficiencies of nitriloside (vitamin B17), folate (vitamin B9), pyridoxal (vitamin B6), and cobalamin (vitamin B12) contribute to abnormal homocysteine metabolism in degenerative diseases. Dietary deficiency of proteins containing sulfur amino acids down-regulates cystathionine synthase, causing abnormal homocysteine metabolism and increased risk of cardiovascular disease. Infections by a variety of micro-organisms promote abnormal homocysteine metabolism in atherogenesis, and carcinogenesis, leading to creation of vulnerable plaques of arteries and dysplastic transformation of susceptible cells of various organs. Neurodegeneration is thought to occur due to similar metabolic derangement. Our protocol to combat hyperhomocysteinemia and subsequent mitochondrial dysfunction includes, thioretinamide, and cobalamin as precursors of thioretinaco, combined with pancreatic enzyme extracts, nutritional modification to eliminate processed foods and to enhance dietary consumption of nitrilosides and other vitamins, and combined with vitamin supplements, essential amino acids, beneficial dietary fats, beneficial dietary protein, and antibiotics to combat chronic infections. The nutritional-metabolic concept of our protocol in SAHACT is adopting intelligent scientific directions incorporating the human cellular pathways in health and disease to combat the pathological process of degenerative disorders, most importantly atherosclerosis and neurodegeneration and cancer. The role of mitochondrial dysfunction in aging and degenerative diseases is key to our understanding of the SAHACT protocol [79]. It is based on the understanding of the function of thioretinamide, thioretinaco, and thioretinaco ozonide in the metabolic origin of degenerative diseases, including arteriosclerosis, dementia and other neurodegenerative diseases ,stroke, acute coronary syndrome, cancer, , autoimmune diseases such as ulcerative colitis, lupus erythematosus, thyroiditis, rheumatoid arthritis and pernicious anaemia, osteoporosis and fracture, venous thrombosis and embolism, retinal vein thrombosis, macular degeneration, hypothyroidism, accelerated aging, renal failure and uremia, diabetes mellitus, metabolic syndrome, severe psoriasis, organ transplantation with therapeutic immune suppression, protein energy malnutrition, familial or spontaneous amyloidosis, dietary deficiencies of folate, pyridoxal, and cobalamin, complications of pregnancy such as placenta previa and pre-eclampsia, and congenital birth defects including neural tube defects, cleft palate, and congenital heart disease. In each of these degenerative diseases and conditions, abnormal homocysteine metabolism has been demonstrated by an increased level of homocysteine bound to plasma proteins by disulphide bonds. The efficacy of this novel nutritional-metabolic approach to prevention and therapy of the important degenerative diseases is rising sun in the horizon. Our innovative protocol carries the optimism of ameliorating and/or aborting the process of atherosclerosis and neurodegeneration in human species.

\section{Other mitochondrial dysfunction innovative therapies}

Beside our nutritional-metabolic therapeutic approach of human degenerative disorders, other therapeutic interventions are emerging in the last few years, including: dietary changes, exercise and medications which target the mechanisms of oxidative stress, inflammation, cardiac hypertrophy, fibrosis and apoptosis [80]. An important consideration in this direction is to understand that common cellular pathways in disease initiation, progression and treatment are common between different degenerative mitochondrial dysfunctions disorders. Hence, specific research in one disease pathology does not deny careful extrapolation of the rapeutic options in clinical trials based on the common potential pathogenicity, namely, the mitochondrial dysfunction. Survival benefit in patients with heart failure has been shown with daily administration of $2 \mathrm{gr}$ of L-carnitine [81]. Daily administration of $9 \mathrm{~g} \mathrm{~L}$-carnitine for a period of 3 months resulted in improvement of mild diastolic dysfunction [82]. Cardiac remodeling is suggested to occur due to administration of L-carnitine through decreased ROS production [83]. Polyphenols such as flavolons, theaflavin and epicatechin present in natural sources such as grape, green tea, olive oil and dark chocolate are known to have important anti-oxidant actions against several chronic diseases including cardiovascular disease [84-86]. For example, quercetin decreases the levels of superoxide and increases urinary excretion of nitrate, 
endothelial NO synthase activity and heme oxygenase-1 protein which has anti-oxidant actions [87]. Moreover, polyphenols of olive oil and red grape reduce intracellular ROS levels [88] whereas epicatechin of green tea lowers the expression of pro-inflammatory molecules [89]. CoQ10 - present in the inner mitochondrial membrane and is important for the production of ATP- possesses anti-thrombotic and anti-oxidant actions and improves hypertension and hyperglycemia [90]. Administration of CoQ10 improved endothelial function and decreased cardiac hypertrophy in hypertensive rats [91]. Supplementation of $100 \mathrm{mg}$ daily CoQ10 for 30 days was found to treat one of the most devastating side effects of statins, that is muscle pain [92]. CoQ10 is considered good therapeutic option for the treatment of mitochondrial dysfunction in humans and can be administrated alone or along with other medications against hypertension and HF although its properties are not fully elucidated in ischemic heart disease [93]. In order to selectively target mitochondria , conjugation to lipophilic molecules is practiced. MitoQ10 improves endothelial NO bioavailability and induce systolic blood pressure reduction by approximately $25 \mathrm{~mm}$ Hg over the 8 week MitoQ 10 treatment period in a model of spontaneously hypertensive rats [91]. MitoQ10 treatment with losartan has revealed beneficial actions against target organ damage development in hypertension [94]. Endogenous inorganic superoxide dismutase (SOD) activity can be mimicked by synthetized molecules such as EUK-8 and EUK-13and have displayed direct mitochondrial anti-oxidant actions against ischemic reperfusion injury [95]. Aerobic exercise was found to increases the production of NO, lowers the levels of superoxide and hydrogen peroxide and improves endogenous enzymatic anti-oxidant systems [96,97]. Aerobic exercise reduces blood pressure in comprehensive modality where systolic and diastolic blood pressure in hypertensive subjects are both reduced in clear comparative advantage over isometric exercise which affects only systolic blood pressure $[98,99]$. Stimulation of NAD+ biosynthetic pathway which increases protein deacetylation through Silent information regulator 1(SIRT1) [100]. Treatment with the NAD+ precursor nicotinamide mononucleotide (NMN) has indicated normalization of NAD+/NADH ratio and protection against diet or age induced diabetes [101]. Silent information regulator 1 (SIRT1) exerts neuroprotection in many neurodegenerative diseases. Peroxisome proliferator-activated receptor gamma( PPAR gamma) coactivator 1 alpha (PGC-1 $\alpha$ ) plays a critical role as a metabolic sensor, which is responsible for the fine-tuning of transcriptional responses to a plethora of stimuli. Activation of SIRT-1-PGC1 $\alpha$ signaling by resveratrol( present in grapes, blueberries, raspberries, mulberries, and peanuts) improves mitochondrial biogenesis [102]. Resveratrol hindered the progression to diabetic cardiomyopathy in animal models [103].

Edaravone which was proved to treat stroke and ALS in Japan, is a novel free radical scavenger which was found useful to reduce pressure overload induced left ventricular hypertrophy in mice through inhibition of Apoptosis Signal regulating Kinase1(Ask1) and its downstream kinases [104] and to reduce perivascular and intermuscular fibrosis and improved cardiac hypertrophy even when treatment was initiated after the onset of cardiac hypertrophy [105]. Elamipretide (SS-31) is a novel, water-soluble tetrapeptide which enhances mitochondrial energy production. Elamipretide binds selectively to cardiolipin and preserves the structure of mitochondrial cristae(which is the main site of oxidative phosphorylation), and the function of oxidative phosphorylation as a whole [106].

\section{Mitochondrial nanomedicine: the future}

Nanotechnology and the recent development of nano particles(NP-based strategies) with its inherent nature to deal with nano size particle carries major hope to dynamite the relatively stagnant therapeutics of subcellular delicate structures like mitochondria and its associated dysfunctional disorders. In our perspective, It is the golden choice for the future dream of personalized "precision medicine". Early advances although promising and encouraging, however, careful examinations of targeting properties of nanomaterials need to be critically explored to take advantage of these unique materials and their properties for subcellular targeting. An example of the workers in the field, $\mathrm{Ru}$ Wen et al. in unique paper on nano medicine and mitochondrial novel therapeutic strategies elaborate extensively in different nano approaches. They document that confocal microscopy imaging of targeted and non-targeted NP treated cells indicated significantly higher association of targeted NPs with the mitochondria of cells whereas the non-targeted NPs were mainly found scattered in the cytosol. The versatility of these NPs was demonstrated by delivering a variety of mitochondria-acting therapeutics, such as, curcumin, mitochondrial decoupler 2,4-dinitrophenol (2,4-DNP) as an antiobesity drug, LND, and $\alpha$-TOS. A significant increase in the potency was observed by directing these therapeutics to the mitochondria [107]. Fluorescence imaging is the evaluating tool for targeting abilities, for most experiments now adyas. Interpretation of mitochondrial association properties of such nano delivery vehicles must be interpreted with caution considering the limitations. Multiple complementary techniques needs to be developed to understand the association properties of delivery vehicles with the mitochondria.

\section{Conclusion}

The science of mitochondrial dysfunction is representing new era of scientific understanding of the deep roots of wide array of human diseases. In the last two decades, the medical literature witnessed the growth of new knowledge of mitochondrial dysfunction as precursore for human degenerative diseases, most 
importantly ; atherosclerosis and neurodegeneration. Most central pathological feature of the neurodegenerative diseases is apoptosis with altered signaling of the apoptotic mechanisms are involved in neurodegeneration. Critical pathologic feature of mitochondrial dysfunction in neurodegeneration is the excess in reactive oxygen species (ROS) production. Raised ROS levels initiates cascade of events leading to the non-apoptotic death of cells. ROS produced in cells acts as signaling molecules, but when produced in abundance will result in decreased effectiveness of cellular mechanisms, initiation of inflammatory pathways, excitotoxicity, protein agglomeration and apoptosis. Mitochondrial dysfunction is precursor for atherosclerosis due to mitochondrial DNA damage, defective oxygen phosphorylation with abundance of ROS, endothelial dysfunction, vascular smooth muscle cells abnormality as well as macrophage activation. The importance of homocysteine in vascular function, arteriosclerosis and neurodegeneration is discussed. Preserving efficient mitochondrial function has been proposed as the most effective therapeutic approach to attenuate the pathogenesis of neurodegenerative diseases, atherosclerosis and other degenerative disorders in human. An innovative unique approach (based on homocysteine theory)targeting mitochondrial apoptotic pathways and oxidative phosphorylation defects and other vital mitochondrial dysfunction consequences is discussed. Nanotechnology and the recent development of nano particles(NPbased strategies) with its inherent nature to deal with nano size particle carries major hope to dynamite the relatively stagnant therapeutics of subcellular delicate structures like mitochondria and its associated dysfunctional disorders. Those innovations are carrying new will toward ameliorating and/or aborting the process of atherosclerosis and neurodegeneration in human species.

\section{References}

1. Sean G Carriedo, Stefano L Sensi, Hong Z Yin, John H (2000) Weiss AMPA exposures induce mitochondrial $\mathrm{Ca}(2+)$ overload and ROS generation in spinal motor neurons in vitro. J Neurosci 20(1): 240-250.

2. De Coo IF, Renier WO, Ruitenbeek W, Ter Laak HJ, Bakker M, et al (1999) A 4-base pair deletion in the mitochondrial cytochrome b gene associated with parkinsonism/MELAS overlap syndrome. Ann Neurol 45(1): 130-133.

3. Coskun PE, Beal MF, Wallace DC (2004) Alzheimer's brains harbor somatic mtDNA control-region mutations that suppress mitochondrial transcription and replication. Proc Natl Acad Sci U S A 101(29): 1072610731.

4. Smigrodzki R, Parks J, and Parker WD (2004) High frequency of mitochondrial complex I mutations in Parkinson's disease and aging. Neurobiol Aging 25(10): 1273-1281.

5. Parker WD Jr and Parks JK (2005) Mitochondrial ND5 mutations in idiopathic Parkinson's disease. Biochem Biophys Res Commun 326(3):667-669.

6. Bender A, Krishnan KJ, Morris CM, Taylor GA, Reeve AK, et al. (2006) High levels of mitochondrial DNA deletions in substantia nigra neurons in aging and Parkinson disease. Nat Genet 38(5): 515-517.

7. Reeve AK, Krishnan KJ, Elson JL, Morris CM, Bender A, et al. (2008) Nature of mitochondrial DNA deletions in substantia nigra neurons. Am J Hum Genet 82(1): 228-235.
8. Puigserver P and Spiegelman BM (2003) Peroxisome proliferatoractivated receptor- gamma coactivator 1 alpha (PGC-1 alpha): transcriptional coactivator and metabolic regulator. Endocr Rev 24(1): 78-90.

9. St-Pierre J, Drori S, Uldry M, Silvaggi JM, Rhee J, et al. (2006) Suppression of reactive oxygen species and neurodegeneration by the PGC-1 transcriptional coactivators. Cell 127(2): 397-408.

10. Abdullah Alabdulgader (2017 )Future of cardiovascular practice: Alert to change or call for revolution. J Cardiovasc Med Ther 1(1): 1.

11. Wenxi Peng, Guoding Cai, Yiping Xia, Jinna Chen, Peng Wu, et al. (2019) Mitochondrial Dysfunction in Atherosclerosis. DNA AND CELL BIOLOGY 38(7): 597-606.

12. Madamanchi N R, Runge M S (2007) Mitochondrial dysfunction in atherosclerosis. Circ Res 100(4): 460-473.

13. Yu E P, Bennett M R (2016) The role of mitochondrial DNA damage in the development of atherosclerosis. Free Radic Biol Med 100: 223-230.

14. Lemieux H, Semsroth S, Antretter H, Hofer D, Gnaiger E, et al. (2011) Mitochondrial respiratory control and early defects of oxidative phosphorylation in the failing human heart. Int J Biochem Cell Biol 43(12): 1729-1738.

15. Madamanchi NR, Runge M S (2007) Mitochondrial dysfunction in atherosclerosis. Circ Res 100(4): 460-473.

16. Yu E P K, Reinhold J, Yu H, Starks L, Uryga A K, et al. (2017) Mitochondrial respiration is reduced in atherosclerosis, promoting necrotic core formation and reducing relative fibrous cap thickness. Arterioscler Thromb Vasc Biol 37(12): 2322-2332.

17. Wang Y, Wang G Z, Rabinovitch P S, Tabas I, (2014) Macrophage mitochondrial oxidative stress promotes atherosclerosis and nuclear factor-kappaB-mediated inflammation in macrophages. Circ Res 114(3): 421-433.

18. Yeh H L, Kuo L T, Sung F C, Yeh C C, (2018) Association between polymorphisms of antioxidant gene (MnSOD, CAT, and GPx1) and risk of coronary artery disease. Biomed Res Int 2018: Pp 5086869.

19. Naik E, Dixit V M (2011) Mitochondrial reactive oxygen species drive proinflammatory cytokine production. J Exp Med 208(3): 417-420.

20. Yu E P K, Reinhold J, Yu H, Starks L, Uryga A K, et al. (2017) Mitochondrial respiration is reduced in atherosclerosis, promoting necrotic core formation and reducing relative fibrous cap thickness. Arterioscler Thromb Vasc Biol 37(12): 2322-2332.

21. Jacinto T A, Meireles G S, Dias A T, Aires R, Porto M L, etal. (2018) Increased ROS production and DNA damage in monocytes are biomarkers of aging and atherosclerosis. Biol Res 51(1): 33.

22. Virag L (2005) Structure and function of poly (ADP-ribose) polymerase-1: role in oxidative stress-related pathologies. Curr Vasc Pharmacol 3(3): 209-214.

23. Mercer J R, Yu E, Figg N, Cheng K K, Prime TA, Griffin J L, et al. (2012) The mitochondria-targeted antioxidant MitoQ decreases features of the metabolic syndrome in ATM+/-/ApoE-/- mice. Free Radic Biol Med 52(5): 841- 849.

24. Salvayre R, Negre Salvayre A, Camare C (2016) Oxidative theory of atherosclerosis and antioxidants. Biochimie 125: 281-296.

25. Victor V M, Apostolova N, Herance R, Hernandez Mijares A, Rocha $M$, et al. (2009) Oxidative stress and mitochondrial dysfunction in atherosclerosis: mitochondria targeted antioxidants as potential therapy. Curr Med Chem 16(35): 4654-4667.

26. Kadlec A O, Chabowski D S, Ait Aissa K, Gutterman D D (2016) Role of PGC-1alpha in vascular regulation: implications for atherosclerosis. Arterioscler Thromb Vasc Biol 36(8): 1467-1474.

27. Pircher A, Treps L, Bodrug N, Carmeliet P (2016) Endothelial cell 
metabolism: a novel player in atherosclerosis? Basic principles and therapeutic opportunities. Atherosclerosis 253: 247-257.

28. Frey R S, Gao X, Javaid K, Siddiqui S S, Rahman A, et al. (2006) Phosphatidylinositol 3-kinase gamma signaling through protein kinase Czeta induces NADPH oxidase-mediated oxidant generation and NFkappaB activation in endothelial cells. J Biol Chem 281(23): 1612816138.

29. Chistiakov D A, Shkurat T P, Melnichenko A A, Grechko A V, Orekhov A $\mathrm{N}$, et al. (2018) The role of mitochondrial dysfunction in cardiovascular disease: a brief review. Ann Med 50(2): 121-127.

30. Guo C, Wang J, Jing L, Ma R, Liu X, et al. (2018) Mitochondrial dysfunction, perturbations of mitochondrial dynamics and biogenesis involved in endothelial injury induced by silica nanoparticles. Environ Pollut 236: 926-936.

31. Serrano M C, Pagani R, Manzano M, Comas J V, Portoles M T, et al. (2006) Mitochondrial membrane potential and reactive oxygen species content of endothelial and smooth muscle cells cultured on poly(epsiloncaprolactone) films. Biomaterials 27(27): 4706-4714.

32. Ruan C, Shen Y, Chen R, Wang Z, Li J, et al. (2013) Endothelial progenitor cells and atherosclerosis. Front Biosci (Landmark Ed) 18; 1194-1201.

33. Szewczyk A, Jarmuszkiewicz W, Koziel A, Sobieraj I, Nobik W, et al. (2015) Mitochondrial mechanisms of endothelial dysfunction. Pharmacol Rep 67(4): 704-710.

34. Gorman GS, Chinnery PF, DiMauro S, etal. (2016) Mitochondrial diseases. Nat Rev Dis Primers 2: 16080

35. Falkenberg M, Hirano M Editing the Mitochondrial Genome. N Engl J Med 383(15): 1489-1491.

36. Hroudová J, Namrata S, Zdenjk Fišar, (2014) Mitochondrial Dysfunctions in Neurodegenerative Diseases: Relevance to Alzheimer's Disease. BioMed Res 2014: 175062.

37. Chen H, McCaffery JM, Chan DC, (2007) Mitochondrial fusion protects against neurodegeneration in the cerebellum. Cell 130(3): 548-562.

38. Swerdlow RH, Burns JM, and Khan SM, (2010) The Alzheimer's disease mitochondrial cascade hypothesis. J Alzheimers Dis 20(2): 265-279.

39. Gibson GE, Sheu KF, Blass JP, (1998) Abnormalities of mitochondrial enzymes in Alzheimer disease. J Neural Transm 105(8-9): 855-870.

40. Manczak M, Park BS, Jung Y, Reddy PH, (2004) Differential expression of oxidative phosphorylation genes in patients with Alzheimer's disease: implications for early mitochondrial dysfunction and oxidative damage. Neuromol Med 5(2): 147-162.

41. Bubber P, Haroutunian V, Fisch G, Blass JP, Gibson GE, (2005) Mitochondrial abnormalities in Alzheimer brain: mechanistic implications. Ann Neurol 57(5): 695-703.

42. Kish SJ, Bergeron C, Rajput A, Dozic S, Mastrogiacomo F, et al. (1992) Brain cytochrome oxidase in Alzheimer's disease. J Neurochem 59(2): 776-779.

43. Bosetti F, Brizzi F, Barogi S, Mancuso M, Siciliano G, et al. (2002) Cytochrome c oxidase and mitochondrial F1F0-ATPase (ATP synthase) activities in platelets and brain from patients with Alzheimer's disease. Neurobiol Aging 23(3): 371-376.

44. Sheng B, Wang X, Su B, Lee HG, Casadesus G, et al. (2012) Impaired mitochondrial biogenesis contributes to mitochondrial dysfunction in Alzheimer's disease. J Neurochem 120(3): 419-429.

45. Narendra DP and Youle RJ, (2011) Targeting mitochondrial dysfunction: role for PINK1 and Parkin in mitochondrial quality control. Antioxid Redox Signal 14(10): 1929-1938.

46. Warby SC, Montpetit A, Hayden AR, Jeffrey BC, Stefanie L, et al. (2009) CAG expansion in the Huntington disease gene is associated with a specific and targetable predisposing haplogroup. Am J Hum Genet 84(3):
$351-366$.

47. Ashu Johri, Flint Beal, (2012) Mitochondrial Dysfunction in Neurodegenerative Diseases. J pharmacol exp ther 342(3): 619-630.

48. Browne SE, Beal MF, (2004) The energetics of Huntington's disease. Neurochem Res 29(3): 531-546.

49. Sasaki S, Horie Y, Iwata M (2007) Mitochondrial alterations in dorsal root ganglion cells in sporadic amyotrophic lateral sclerosis. Acta Neuropathol 114(6): 633-639.

50. Keep M, Elmer E, Fong KS, Csiszar K (2001) Intrathecal cyclosporin prolongs survival of late-stage ALS mice. Brain Res 894(2): 327-331.

51. Mattiazzi M, D’Aurelio M, Gajewski CD, Martushova K, Kiaei M, etal. (2002) Mutated human SOD1 causes dysfunction of oxidative phosphorylation in mitochondria of transgenic mice. J Biol Chem 277(33): 29626-29633.

52. Menzies FM, Cookson MR, Taylor RW, Turnbull DM, Chrzanowska L, et al. (2002) Mitochondrial dysfunction in a cell culture model of familial amyotrophic lateral sclerosis. Brain 125(7): 1522-1533.

53. Beal MF, Ferrante RJ, Browne SE, Matthews RT, Kowall NW, et al. (2008) Mitochondrial fragmentation in neurodegeneration. Nat Rev Neurosci 9(7): 505-518.

54. Beal MF, Ferrante RJ, Browne SE, Matthews RT, Kowall NW, et al. (1997) Increased 3-nitrotyrosine in both sporadic and familial amyotrophic lateral sclerosis. Ann Neurol 42(4): 644-654.

55. Beal MF (2002) Review Oxidatively modified proteins in aging and disease. Free Radic Biol Med 32(9): 797-803.

56. Szewczyk A, Jarmuszkiewicz W, Koziel A, Sobieraj I , Nobik W, et al. (2015) Mitochondrial mechanisms of endothelial dysfunction. Pharmacol Rep 67(4): 704-710.

57. Frey R S, Gao X, Javaid K, Siddiqui SS, Rahman A, et al. (2006) Phosphatidylinositol 3-kinase gamma signaling through protein kinase Czeta induces NADPH oxidase-mediated oxidant generation and NFkappaB activation in endothelial cells. J Biol Chem 281(23): 1612816138.

58. Kluge MA, Fetterman JL, Vita JA (2013) Mitochondria and endothelial function. Circ Res 112(8): 1171-1188.

59. Won JC, Park JY, Kim YM, Koh EH, Seol S, et al. (2010) Peroxisome proliferator-activated receptor gamma coactivator 1-alpha overexpression prevents endothelial apoptosis by increasing ATP/ADP translocase activity. Arterioscler Thromb Vasc Biol 30(2): 290-297.

60. Stein S, Lohmann C, Handschin C, Stenfeldt E, Boren J, et al. (2010) ApoE/- PGC-1alpha-/- mice display reduced IL-18 levels and do not develop enhanced atherosclerosis. PLoS One 5(10): e13539.

61. Oldendorf WH, Cornford ME, Brown WJ (1977) the large apparent work capability of the blood-brain barrier: a study of the mitochondrial content of capillary endothelial cells in brain and other tissues of the rat. Ann Neurol 1(5): 409-417

62. Tretter L, Ambrus A (2014) Measurement of ROS homeostasis in isolated mitochondria. Methods Enzymol 547: 199-223.

63. Sun Q Zhong W, Zhang W, Zhou Z (2016) Defect of mitochondrial respiratory chain is a mechanism of ROS overproduction in a rat model of alcoholic liver disease: role of zinc deficiency. Am J Physiol Gastrointest Liver Physiol 310(3): G205-G214.

64. Durham AL, Speer MY, Scatena M, Giachelli CM, Shanahan CM, et al. (2018) Role of smooth muscle cells in vascular calcification: implications in atherosclerosis and arterial stiffness. Cardiovasc Res 114(4): 590-600.

65. Chiong M, Cartes Saavedra B , Norambuena Soto I, Mondaca Ruff D, MoralesPE, et al. (2014) Mitochondrial metabolism and the control of vascular smooth muscle cell proliferation. Front Cell Dev Biol 2: 72. 
66. Docherty CK, Carswell A, Friel E, Mercer JR (2018) Impaired mitochondrial respiration in human carotid plaque atherosclerosis: a potential role for Pink1 in vascular smooth muscle cell energetics. Atherosclerosis 268: 1-11.

67. Colin S, Chinetti Gbaguidi G, Staels B (2014) Macrophage phenotypes in atherosclerosis. Immunol Rev 262(1): 153-166.

68. Chinetti Gbaguidi G, Colin S, Staels B (2015) Macrophage subsets in atherosclerosis. Nat Rev Cardiol 12: 10-17.

69. Van den Bossche J, Baardman J, Otto NA, van der Velden S, Neele AE, et al. (2016) Mitochondrial dysfunction prevents repolarization of inflammatory macrophages. Cell Rep 17(3): 684-696.

70. Stoger JL, Gijbels MJ, van der Velden S, Manca M, van der Loos CM, et al. (2012) Distribution of macrophage polarization markers in human atherosclerosis. Atherosclerosis 225(2): 461-468.

71. Vats D, Mukundan L, Odegaard JI, Zhang L, Smith KL, et al. (2006) Oxidative metabolism and PGC- 1 beta attenuate macrophage-mediated inflammation. Cell Metab 4(1): 13-24.

72. Van den Bossche J, Baardman J, Otto NA, van der Velden S, Neele AE, et al. (2016) Mitochondrial dysfunction prevents repolarization of inflammatory macrophages. Cell Rep 17(3): 684-696.

73. Sica A, Mantovani A (2012) Macrophage plasticity and polarization: in vivo veritas. J Clin Invest 122(3): 787-795.

74. Boyle JJ, Johns M, Kampfer T, Nguyen AT, Game L, et al. (2012) Activating transcription factor 1 directs Mhem atheroprotective macrophages through coordinated iron handling and foam cell protection. Circ Res 110(1): 20-33.

75. Alabdulgader A, Kilmer McCully, Paul Rosch Saudi Arabia Homocysteine Arteriosclerosis Cancer Trial (SAHACT) basic proposal .ASTP-09. ADVANCED AND STRATEGIC TECHNOLOGIES.KACST.

76. Seshadri S, Beiser A, Selhub J, Jacques PF, Rosenberg IH, et al.(2002) Plasma homocysteine as a risk factor for dementia and Alzheimer's disease. New Engl J Med 346(7): 476-483.

77. Levine J, Stahl Z, Sela BA, Gavendo S, Ruderman V, et al.(2002) Elevated homocysteine levels in young male patients with schizophrenia. Amer J Psychiatry 159(10): 1790-1792.

78. Morris MS, Jacques PF, Rosenberg IH, Selhub J(2001) Hyperhomocysteinemia associated with poor recall in the Third National Health and Nutrition Examination Survey. Amer J Clin Nutr 73(5): 927-933.

79. Russo C, Morabito F, Luise F, Piromali A, Battaglia L, et al. (2008) Hyperhomocysteinemia is associated with cognitive impairment in multiple sclerosis. J Neurol 255(1): 64-69.

80. Towards Revolutionary Understanding of Cellular Pathways in Health and Disease: Homocysteine Metabolism, Mitochondrial Dysfunction and the SAHACT Trial'. Abdullah A Alabdulgader, Kilmer S. McCully, Paul J. Rosch Lipid Lunacy: Diet Delusions and what really causes heart disease. Published by Columbus Publishing Ltd 2020. ISBN: 9781907797736

81. Siasos G, Tsigkou V, Kosmopoulos M, Theodosiadis D, Simantiris $S$, et al. (2018)Mitochondria and cardiovascular diseases-from pathophysiology to treatment. Ann Transl Med 6(12): 256

82. Rizos I (2000) Three-year survival of patients with heart failure caused by dilated cardiomyopathy and L-carnitine administration. Am Heart 139(2 pt 3): S120-S123.

83. Serati AR, Motamedi MR, Emami S (2010) L-carnitine treatment in patients with mild diastolic heart failure is associated with improvement in diastolic function and symptoms. Cardiology 116(3): 178-182.

84. O’Brien D, Chunduri P, Iyer A (2010) L-carnitine attenuates cardiac remodelling rather than vascular remodelling in deoxycorticosterone acetate-salt hypertensive rats. Basic Clin Pharmacol Toxicol 106(4):
296-301.

85. Kokkou E, Siasos G, Georgiopoulos G (2016) the impact of dietary flavonoid supplementation on smoking-induced inflammatory process and fibrinolytic impairment. Atherosclerosis 251: 266-272.

86. Siasos G, Tousoulis D, Kokkou E (2014) Favorable effects of Concord grape juice on endothelial function and arterial stiffness in healthy smokers. Am J Hypertens 27: 38-45.

87. Siasos G, Tousoulis D, Tsigkou V (2013) Flavonoids in atherosclerosis: an overview of their mechanisms of action. Curr Med Chem 20(21): 2641 2660

88. Loke WM, Proudfoot JM, Hodgson JM (2010) Specific dietary polyphenols attenuate atherosclerosis in apolipoprotein E-knockout mice by alleviating inflammation and endothelial dysfunction. Arterioscler Thromb Vasc Biol 30(4): 749-757.

89. Scoditti E, Calabriso N, Massaro M(2012) Mediterranean diet polyphenols reduce inflammatory angiogenesis through MMP-9 and COX-2 inhibition in human vascular endothelial cells: a potentially protective mechanism in atherosclerotic vascular disease and cancer. Arch Biochem Biophys 527(2): 81-89.

90. Natsume M, Baba S (2014) Suppressive effects of cacao polyphenols on the development of atherosclerosis in apolipoprotein E-deficient mice. Subcell Biochem 77: 189-198.

91. Garrido Maraver J, Cordero MD, Oropesa Avila M (2014) Clinical applications of coenzyme Q10. Front Biosci (Landmark Ed) 19: 619-633.

92. Graham D, Huynh NN, Hamilton CA (2009) Mitochondria-targeted antioxidant MitoQ10 improves endothelial function and attenuates cardiac hypertrophy. Hypertension 54(2): 322-328.

93. Littarru GP, Langsjoen P (2007) Coenzyme Q10 and statins: biochemical and clinical implications. Mitochondrion 7 Suppl: S168-S174.

94. Pepe S, Marasco SF, Haas SJ (2007) Coenzyme Q10 in cardiovascular disease. Mitochondrion 7 Suppl: S154-S167.

95. McLachlan J, Beattie E, Murphy MP (2014) Combined therapeutic benefit of mitochondria-targeted antioxidant, MitoQ10, and angiotensin receptor blocker, losartan, on cardiovascular function. J Hypertens 32(3): 555-564

96. Pucheu S, Boucher F, Sulpice T (1996) EUK-8 a synthetic catalytic scavenger of reactive oxygen species protects isolated iron-overloaded rat heart from functional and structural damage induced by ischemia/ reperfusion. Cardiovasc Drugs Ther 10(3): 331-339.

97. Skrypnik D, Bogdanski P, Madry E(2014) Effect of physical exercise on endothelial function, indicators of inflammation and oxidative stress. Pol Merkur Lekarski 36(212): 117-121.

98. Roberts CK, Won D, Pruthi S (2006) Effect of a diet and exercise intervention on oxidative stress, inflammation and monocyte adhesion in diabetic men. Diabetes Res Clin Pract 73(3): 249-259.

99. Korsager Larsen M, Matchkov VV (2016) Hypertension and physical exercise: The role of oxidative stress. Medicina (Kaunas) 52(1): 19-27.

100. Siasos G, Athanasiou D, Terzis G(2016) Acute effects of different types of aerobic exercise on endothelial function and arterial stiffness. Eur J Prev Cardiol 23(14): 1565-1572.

101. Wang G, Han T, Nijhawan D (2014) P7C3 neuroprotective chemicals function by activating the rate-limiting enzyme in NAD salvage. Cell 158(6): 1324-1334.

102. Yoshino J, Mills KF, Yoon MJ (2011) Nicotinamide mononucleotide, a key $\mathrm{NAD}(+)$ intermediate, treats the pathophysiology of diet- and ageinduced diabetes in mice. Cell Metab 14(4): 528-536.

103. Chan V, Fenning A, Iyer A (2011) Resveratrol improves cardiovascular function in DOCA-salt hypertensive rats. Curr Pharm Biotechnol 12: 
429-436.

104. Thirunavukkarasu M, Penumathsa SV, Koneru S (2007) Resveratrol alleviates cardiac dysfunction in streptozotocin-induced diabetes: Role of nitric oxide, thioredoxin, and heme oxygenase. Free Radic Biol Med 43(5): 720-729.

105. Date MO, Morita T, Yamashita N (2002) the antioxidant N-2 mercaptopropionyl glycine attenuates left ventricular hypertrophy in in vivo murine pressure-overload model. J Am Coll Cardiol 39(5): 907-912.

106. Tsujimoto I, Hikoso S, Yamaguchi O (2005) the antioxidant edaravone attenuates pressure overload-induced left ventricular hypertrophy. Hypertension 45(5): 921-926.

107. Szeto HH (2014) First-in-class cardiolipin-protective compound as a therapeutic agent to restore mitochondrial bioenergetics. $\mathrm{Br}$ Pharmacol 171(8): 2029-2050.
108. RuWen, Bhabatosh Banik, Anil Kumar, Nagesh Kolishetti, Shanta Dhar, et al.(2016)Nanotechnology inspired tools for mitochondrial dysfunction Related diseases, Advanced Drug Delivery Reviews 99(pt A): 52-69. 